\title{
Techniques for Using a Novel Intramedullary Cage Technology for Fixation of Proximal Humeral Fractures
}

\author{
Paul D Paterson' ${ }^{1}$, Eric W Fulkerson' ${ }^{2}$, Andrew K Palmer ${ }^{3 *}$ \\ ${ }^{1}$ Excelsior Orthopedics, Buffalo, New York; \\ ${ }^{2}$ Muir Orthopedic Specialists, Walnut Creek, California; \\ ${ }^{3}$ Professor Emeritus, SUNY Upstate Medical Center, Syracuse, New York 13202
}

Received: August 26, 2016; Accepted: September 19, 2016; Published: September 27, 2016

*Corresponding author: Andrew K Palmer MD, Professor Emeritus, SUNY Upstate Medical Center, Institute for Human Performance-3215, 750 East Adams St, Syracuse NY 13202, Tel: (315) 447 7991; Email: palmerak46@gmail.com

\begin{abstract}
Proximal humeral fragility fractures in the elderly are common Locking plate technology has made stable anatomic reconstruction theoretically possible. However, current surgical techniques using locked plates are associated with unacceptably high complication rates. Since no surgical treatment has distinguished itself, there is no agreed upon preferred treatment option. In this context, a novel intramedullary fracture fixation technology is discussed with the intent of reducing or eliminating the complication concerns with current technologies. This technology - Proximal Humeral (PH) Cage - is indicated for the treatment of 2-, 3-, and 4- part proximal humeral fractures. Two different interventional techniques - a deltopectoral surgical approach and a minimally invasive surgical approach - are detailed in this paper. Our early experience with a human case is provided to illustrate the techniques for fracture reduction and fixation using the PH Cage.
\end{abstract}

Keywords: Proximal Humeral Fracture; Intramedullary Fixation; PH Cage

\section{Introduction}

Proximal humeral fractures account for approximately $6 \%$ of all fractures in adults[1]. Fractures in younger patients are generally a result of high energy trauma such as a consequence of automobile collisions or fall from heights. In elderly patients, minor trauma such as ground level falls result in osteoporotic fractures. Patients with poor quality bone and osteoporosis are at the highest risk of sustaining such fragility fractures[1,2]. Proximal humeral fractures are approximately 3 times more prevalent in women than men[2]. A three-fold increase of these fractures is expected by 2030 when compared to the incidences in 1998[3]. Thus, it is critical to define techniques and develop technologies for optimal management of humeral fractures.

Options for surgical management of proximal humeral fractures have evolved continuously. Traditionally, a majority of these fractures (approximately 80\%) were treated non operatively since the fractures were non-displaced or in consideration of patient age and functional level[4]. However, surgical treatment of these fractures has significantly increased in the US when comparing data from 1999-2000 to 20042005. The preference for surgical treatments has evolved with technological advances especially locking plate technology[4]. Although locking plate technology has become very popular, results are still unpredictable and complication rates remain high[5]. Hence, many surgeons resort toan arthroplasty, which is both costly and oftentimes without a solution for revision should a complication occur. For this reason, an alternative method of fixation of proximal humeral fractures is needed. This paper describes two techniques using a novel implant to treat proximal humeral fractures i.e. the PH Cage technology (Conventus Orthopedics, Maple Grove, MN).

\section{Anatomic Considerations}

Techniques to treat a proximal humeral fracture must consider the anatomy, especially its impact on the musculature, vasculature, and nerves. The supraspinatus, infraspinatus, and teres minor muscles attach to the greater tuberosity. These muscles primarily are responsible for external rotation and abduction forces in the shoulder joint. The subscapularis attaches to the lesser tuberosity and exerts internal rotation forces[6]. In addition to these primary muscles, the other shoulder muscles include the deltoid, pectoralis major, and latissimus dorsi muscles - all of which insert distal to the tuberosities.

The primary blood source for the proximal humerus is believed to be the anterior humeral circumflex artery, which arises from the axillary artery[7,8]. It runs parallel to the lateral aspect of the biceps tendon and enters the bone at the proximal end of the intertubercular groove. The posterior portion of the greater tuberosity and the posterioinferior humeral head are per fused by the posterior humeral circumflex artery. An adequate blood supply to the affected fragments is necessary for fracture healing as well as to minimize the risk of Avascular Necrosis (AVN). Recently, it was determined that the posterior humeral 
circumflex artery is a more prominent source of blood supply to the humeral head than previously recognized[9]. This may explain why risk of AVN is not directly predictable from the disruption of the anterior circumflex artery[10]. Other arteries that provide blood supply to the proximal humerus include the profunda brachii, suprascapular, thoracoacromial, and subscapular arteries[8].

The axillary nerve and the suprascapular nerve are occasionally damaged during proximal humeral fractures. The anterior section of the axillary nerve courses around the humeral shaft approximately $35 \mathrm{~mm}$ from the lateral prominence of the greater tuberosity[11], and is therefore susceptible to injury due to its closeness to fracture fragmentation[12]. During surgical intervention, care must be taken to protect the integrity of the axillary nerve. As the nerve can be reliably palpated as a cord like structure deep to the deltoid muscle, injury is typically rare during surgical interventions[13].

\section{Methods \& Materials}

The PH Cage is an expandable intramedullary nitinol implant intended for fixation of proximal humeral fractures. It is indicated for fixation of 2-, 3-, and 4-part fractures except in instances where extensive fracture comminution may limit fixation options. It is intended to provide internal buttress to the humeral head and medial calcar once reduction is obtained through normal surgical practice. The PH Cage can be inserted using a small entry hole on the bone, thus limiting soft tissue stripping. It is deployed directly below the humeral head particular surface to provide stability and load bearing during healing. The PH Cage allows the surgeon discretion as to the number and direction of screws to reduce and fix the fracture fragments. Currently, the PH Cage can be implanted using two different techniques - a deltopectoral surgical approach or a minimally invasive surgical approach.

\section{Deltopectoral Approach}

A deltopectoral approach is the most commonly used surgical incision for the reduction and fixation of proximal humerus fractures[14]. The patient can be positioned in a beach-chair position or a supine position depending on surgeon preference. The C-arm is positioned such that Anterio Posterior (AP) and lateral views of the shoulder can be obtained intraoperatively.

A 10 to $15 \mathrm{~cm}$ long surgical incision is started at the tip of the coracoid process and runs laterally in the direction of the insertion of the deltoid muscle. The incision is usually maintained medial to the cephalic vein. The clavipectoral fascia is opened, and the tendon is retracted medially following the incision. This technique is used to expose the fracture by using internal and external rotation of the humerus. If necessary, the integrity of the axillary nerve can be confirmed by running the tug test at the inferior border of the subscapularis and beneath the deltoid[15]. The rotator cuff interval may be incised at the level of the penetration of the biceps tendon to mobilize the tuberosity fragments and to view the particular surfaces. Care should be taken so as to not damage the anterior humeral circumflex artery.

The PH Cage is accompanied with instrumentation to aid with reduction and fixation of fractures. A jig, which looks like a locking plate, is used as a template to buttress and reduce the fracture. It is placed lateral to the bicipital groove approximately $10 \mathrm{~mm}$ distal to the apex of greater tuberosity. Standard K-wires (threaded and non-threaded) are used to reduce the fracture fragments to the template jig (Figure 1).

Once the fragments are reduced, an $8 \mathrm{~mm}$ hole is drilled from the distal end of the jig to approximately $5 \mathrm{~mm}$ below the humeral head particular surface. Guide bushings aid with positioning the implant medial, central or lateral depending on the fracture fragmentation. A site preparation tool is then used to create a space for the $\mathrm{PH}$ cage in the sub cortical region below the humeral head (Figure 2). The K-wire holes in the template jig are designed to allow for use of the site preparation device and the implantation of the Cage without interference and/or loss of reduction.

The PH Cage comes preloaded into a delivery tool. This is inserted at the site of the access hole and the cage is deployed below the humeral head at the site prepared by the site preparation tool. The cage is then locked in its expanded state. Implant length and diameter are determined by the patient anatomy, and is chosen from one of three implant sizes available. $3.5 \mathrm{~mm}$ cannulated screws are then used to hold and secure the fracture fragments onto the PH Cage (Figure 3). The surgeon has discretion in the number and direction of screws needed to fix

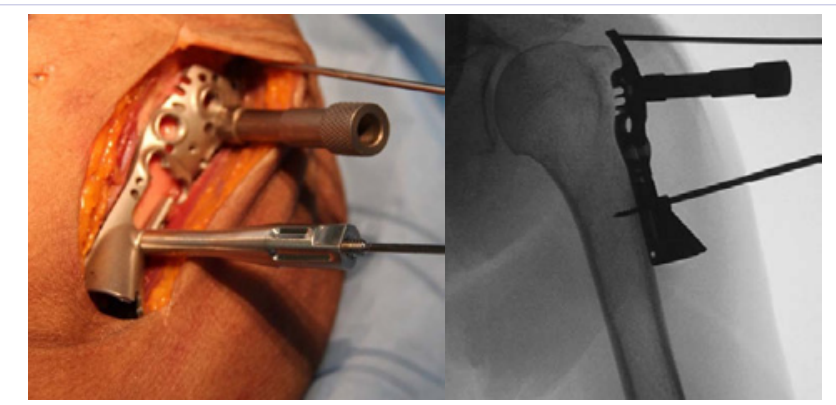

Figure 1: (Left) Jig used to aid fracture reduction and fixation following a deltopectoral incision in a cadaver. (Right) AP view of the jig as it is secured on to the lateral side of the proximal humerus.

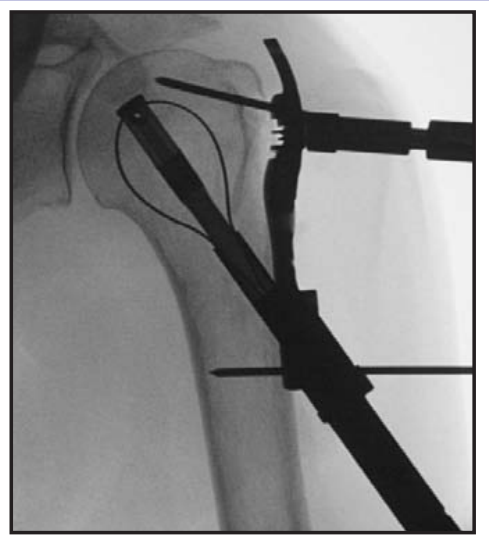

Figure 2: AP view of the site preparation tool used to create a spot for the PH Cage implantation below the humeral head. 
the fracture. Screws extend from the tuberosity to the humeral shaft distally to stabilize the fracture construct. 2- part fractures are adequately secured with one screw in each tuberosity. When the tuberosities are displaced, two screws should be used in each to secure. Titanium washers can be placed on the screws to augment as well as to tie the rotator cuff muscles to the PH Cage. An optional plate is available for use in combination with the $\mathrm{PH}$ Cage if preferred.

Post-operative treatment varies as a result of the severity of the initial injury, the patient's age and overall health, bone quality. Oftentimes, patients treated for 2 part fractures are given a sling for comfort, and allowed to use the arm ad lib. Rehabilitation protocols are started after the first post op visit at 10 days. Multipart fractures are placed in a sling and do gentle pendulums for 10 days. Gentle passive and active assisted exercises begin at 10 days if tolerated. Active motion for these patients is begun at 4-6 weeks post operation.

\section{Minimally Invasive Surgical Approach}

The PH Cage can also be implanted via a minimally invasive surgical approach. This approach provides a closed reduction and fixation of fractures, thus minimizing soft tissue stripping and preserving periosteal blood supply. Indications for use include all 2- part fractures; 3- and 4- part fractures with minimally comminuted greater tuberosity fragments within 5 days of injury. Multi fragment fractures treated beyond 5 days from injury are very difficult to reduce and are better approached through a deltopectoral approach.

The patient can be positioned in a beach-chair position or a supine position depending on surgeon preference. The C-arm is positioned to ensure that AP and lateral views of the shoulder are available intraoperatively. Axillary nerve injury is a greater concern using a minimally invasive surgical approach than a deltopectoral approach. A good minimally invasive surgical technique is essential to avoid axillary nerve injury. The Axillary Nerve Zone (AZN) is a $2 \mathrm{~cm}$ strip along the lateral arm centered $3.5 \mathrm{~cm}$ from the greater tuberosity. When in the ANZ, sharp dissection should only be through skin and subcutaneous tissues. Bluntly dissect down to bone in this zone. All wires, drills, and screws placed in the ANZ should be done through cannulas that are confirmed to rest on the bone using fluoroscopy.

Jigs and instrumentation are available to aid with minimally invasive reduction and fixation of fractures. Surgical markers are used to identify the humeral shaft by tracing a line from the center of the anterolateral acromion to the lateral epicondyle. The foot of the jig is positioned approximately $1 \mathrm{~cm}$ below the greater tuberosity. At this position, the distal end of the jig is located to begin a skin incision. This incision should be over the proximal portion of the broad deltoid insertion, and distal to the axillary nerve. An incision of approximately $3-4 \mathrm{~cm}$ in length is created at the base of the jig to begin the procedure (Figure 4). The skin and subcutaneous tissues are sharply divided. The deltoid insertion is sharply divided longitudinally. A circumferential release of soft tissue from the proximal humerus is performed with a Cobb elevator. Fluoroscopy is useful to ensure the Cobb stays on bone and is not trapped in fracture fragments or soft tissue. The release should extend to the greater tuberosity proximally, and lesser tuberosity anteriorly. The axillary nerve can often be palpated by placing the index finger in the sub deltoid space with the thumb on the skin and the drawing this 'pinch' distally. The axillary nerve can be felt rolling between the pinch. The biceps tendon and lesser tuberosity are also palpable through this incision.

The foot of the jig is inserted through the skin incision and moved to the greater tuberosity. Fluoroscopy is used to insure that the jig stays on the bone, thus preventing axillary nerve entrapment. The jig is first fixed the lateral humeral shaft using a threaded pin and handle. Fracture fragments are reduced using joy sticks, or by working through stab incisions ('arthroscopy portals'). Bone tamps and elevators are used to maneuver fragments. Fragments are provisionally fixed by placing K-wires through the jig. The holes of the jig are designed to hold numerous fragments without interfering with the deployment and use of the PH Cage (Figure 5). Once the fragments are reduced, an $8 \mathrm{~mm}$ hole is drilled from the distal end of the jig to approximately $5 \mathrm{~mm}$ below the humeral head particular surface. A site preparation tool is used create a site for the PH cage in the sub cortical region below the humeral head (Figure 2). Implant deployment and fracture fixation is achieved similar to steps described for the deltopectoral approach previously. Screws are inserted using

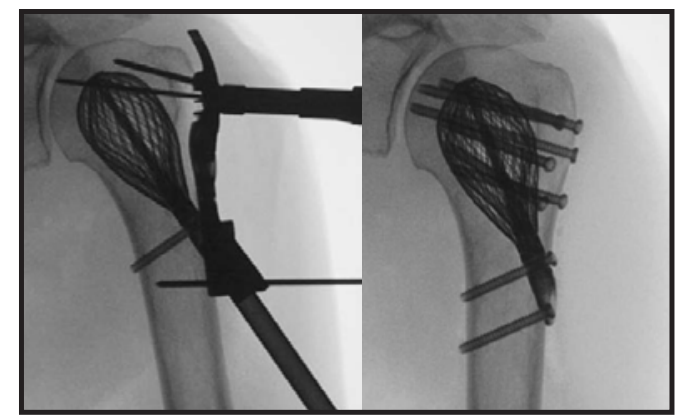

Figure 3: (Left) Deployment of the PH Cage at the site prepared by the site preparation device. Note - a jig aids with holding the reduction in place during PH Cage deployment. (Right) Final construct with the PH Cage and screws. The number and direction of screws to use is at the discretion of the surgeon.

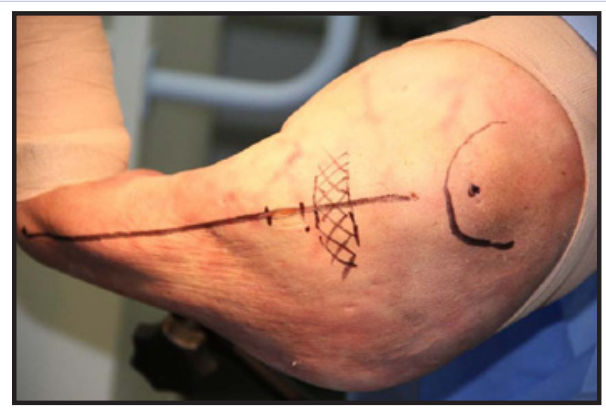

Figure 4: Use of surgical markers to identify the location of the anterolateral acromion and the lateral epicondyle to mark the location of the humeral shaft. The zone where the axillary nerve is expected is marked prior to surgical incision. The location of the incision is also marked. 
small stab incisions and tissue protectors to minimize soft tissue stripping and potential for nerve damage.

Post operatively patients treated for 2 part fractures are given a sling for comfort, and allowed to use the arm ad lib. Rehabilitation protocols are started after the first post op visit at 10 days. Multipart fractures are placed in a sling and do pendulums only for 10 days. Gentle passive and active assisted exercises begin at 10 days. Active motion for these patients is started at 4-6 weeks post operation.

\section{Early Clinical Experience}

A clinical experience using the PH Cage for fracture fixation is discussed. The patient suffered a 2-part fracture from a low energy impact, and the humeral head was in valgus alignment. The minimally invasive surgical approach instrumentation was used per the technique described previously to reduce and fix the fracture. As shown in Figure 6, the fracture was reduced and fixed successfully using a PH Cage and 4 screws. This was accomplished through small incisions requiring minimal surgical morbidity. Early range of motion and rehabilitation protocols have been

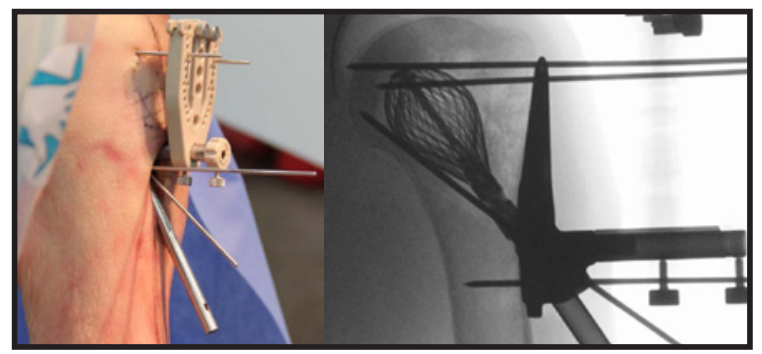

Figure 5: (Left) Jig used for reduction and fixation of proximal humeral fracture using the PH Cage. (Right) AP view of the deployment of the implant. Note that the foot of the implant rests on the surface of the bone and provides a template for fracture reduction.

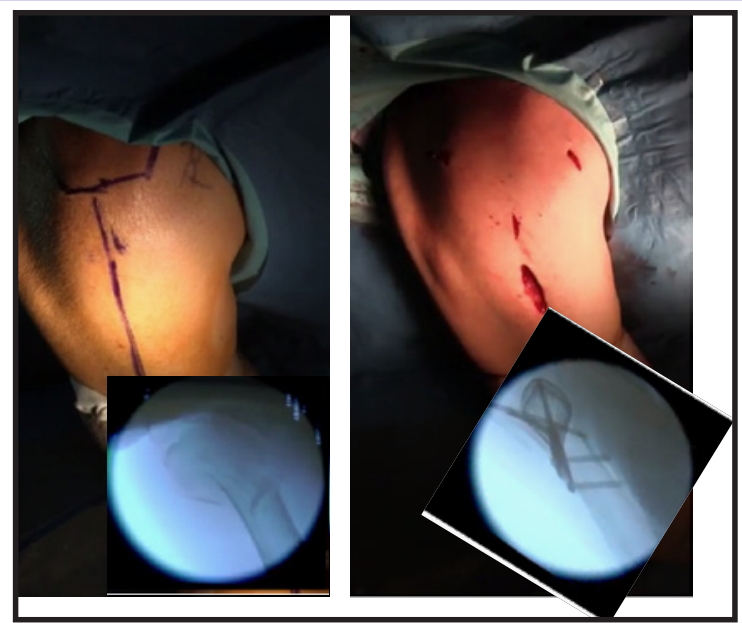

Figure 6: (Top) A patient with a 2-part valgus fracture is readied for treatment with a PH Cage using the minimally invasive surgical approach. (Bottom) Successful fixation of the fracture using the PH Cage and screws. Note that the final incision on the patient is noticeably smaller when compared to a deltopectoral surgical incision (refer Figure 1). used to enhance early functional recovery and improve potential for fracture healing.

\section{Discussion}

Long term clinical outcomes indicate that there is no significant difference in patient quality of life for non-operative vs. surgical management of non-displaced and 2- part fractures[16]. However, early on in the healing phase, patients may experience considerable pain as well as stiffness of the shoulder. As a result, there has been a steady increase in the number of surgical interventions especially with the advent of new technologies (e.g. Locking plates, reverse shoulder arthroplasty)[4]. Surgical intervention is targeted to reduce all the fracture fragments, reestablish the neck-shaft angle, restore the medial calcar, and provide stable fixation. Functional outcomes are dependent on the fracture type and displacement and its subsequent reduction and stabilization as well as the patient age, bone density and patient compliance to treatment regimen[17]. Current options for surgical interventions include percutaneous pinning, intramedullary nails, Open Reduction and Internal Fixation (ORIF) using locking plates, and arthroplasty (hemi or reverse shoulder).

Even the most meticulous surgical technique creates injury. Surgical intervention can cause pain and inflammation that delays and/or limits recovery. Minimizing such injury has stimulated minimally invasive surgical technique development revolutionizing carpal tunnel, rotator cuff, and anterior cruciate ligament surgery to name a few $[18,19]$. Percutaneous techniques for simple proximal humeral fracture fixation have been reported extensively. Currently, these techniques are advised for treatment of 2- part fractures as the outcomes are generally poor when extended to displaced three and four part fractures[20]. These techniques are technically demanding to use and offer less stability when compared to other fixation technologies. Reported complications include high rates of malunion, migration of the pins, insufficient construct stability to support early functional recovery, infection, and osteonecrosis[21].

Intramedullary nails are used for fixation of 2-, 3- and 4- part proximal humerus fractures with minor displacement of the tuberosities. A significant advantage of using intramedullary nails is reduced soft tissue stripping[12]. Though nails are indicated for use with most fracture types, its best functional outcomes are reported for use with 2- part fractures. When compared to locking plates, a prospective randomized trial showed no difference in clinical outcomes at 3 years for patients with 2part fractures [22]. Complications increase when nails are used for fixation of 3- and 4- part fractures with re-intervention rates reported as high as $45 \%$ [23]. Specific complications reported for use of intramedullary nail include screw penetration, nail migration, malunion, and rotator cuff injury resulting in pain to the patients[24].

ORIF of proximal humerus fractures using locking plate technologies are steadily increasing as technologies and techniques evolve. Locking plates are indicated for 2-, 3- and 4- part fractures with a few exceptions where a prosthetic 
replacement may be needed. A deltopectoral surgical incision is most commonly used for such ORIF procedures as it provides direct visualization of the fracture fragments[14]. Buttressing the medial calcar is critical to fracture stability following fixation[25]. Locking plates allow for the use of a kickstand screw to buttress the medial calcar. Fibular strut grafts have been used in conjunction with locking plates to support the medial calcar and have been shown to reduce the incidence of screw penetration or hardware issues [26]. Additionally, plates have eyelets to suture and tie the rotator cuff muscles [27]. However, current surgical techniques using locking plates are associated with unacceptable complication rates. Overall complication rate as high as $48 \%$ is reported from a comprehensive review of literature along with a revision rate of $14 \%$ [28]. Complications include screw perforation of the humeral head, osteonecrosis, mal-union or loss of reduction especially in varus fractures, infection due to soft tissue stripping, and subacromial impingement $[29,30]$.

Management of complex 4- part fractures in osteoporotic patients is possible using ORIF or arthroplasty (hemi or reverse shoulder). Its optimal management is controversial as clinical studies are inconclusive when the two treatment options are compared[31]. Ultimately, the degree of underlying shoulder pathology affects the surgeons' choice of ORIF vs. arthroplasty for elderly patients. Complications that are reported following arthroplasty include shoulder stiffness, glenoid erosion, instability, and scapular notching amongst a host of other minor long term effects[32].

The PH Cage, as introduced in this paper, provides treatment options that should reduce complications noted with current fracture fixation technologies. The implant acts as a buttress to support the medial calcar, and its location beneath the particular surface of the humeral head allows it to support loads during rehabilitation (Figure 3). The benefits of having a medial column support, such as an intramedullary fibular graft, have previously been reported. Specifically, patients treated with an intramedullary fibular strut graft did not have any screw penetration and had high clinical outcome scores $[12,26]$. In addition, the protrusion of the Cage outside the bone is minimal (Figure 3). Since any minimal protrusion of the cage is distal to the greater tuberosity, there is no concern for potential acromial impingement, which is a known issue with locking plate technologies[29].

The use of an intramedullary Cage (DRS Cage) for the fixation of distal radius fractures has previously been reported with good clinical outcomes [33]. That report also indicated early fracture healing was seen, which may have been the result of the pluripotent bone slurry that is created with the use of the site preparation tool for the cage. As the PH Cage is used more and more clinically, attention will be paid to this potential benefit from use of the Conventus Cage.

\section{Summary}

Surgical management of proximal humeral fractures remains a challenge for all types of fractures because of the problems of screw penetration of the humeral head, non-union, loss of intraoperative reduction and hardware failure with the use of locking plates and nails. In an attempt to lessen these complications, the PH Cage has been introduced. This paper details the current state of knowledge of treatment of proximal humeral fractures and outlines our technique of inserting the $\mathrm{PH}$ Cage through a standard deltopectoral incision or alternatively through a minimally invasive technique.

\section{Declarations}

All author(s) are paid consultants associated with Conventus Orthopedics. The authors declare that no benefits have been received or will be received related directly or indirectly to the subject of this article.

\section{References}

1. Court-Brown CM \& Caesar B. Epidemiology of adult fractures: A review. Injury. 2006;37(8):691-697. DOI:10.1016/j.injury.2006.04.130.

2. Baron J, Barrett J \& Karagas M. The epidemiology of peripheral fractures. Bone. 1996;18(3 Supplement):209S-213S.

3. Kannus P, Palvanen M, Niemi S, Parkkari J, Jarvinen M, Vuori I. Osteoporotic fractures of the proximal humerus in elderly Finnish persons: Sharp increase in 1970-1988 and alarming projections for the new millennium. Acta Orthopaedica Scandinavica. 2000;71(5):465470. DOI:10.1080/000164700317381144.

4. Bell J-E, Leung BC, Spratt KF, Koval KJ, Weinstein JD, Goodman DC, et al. Trends and variation in incidence, surgical treatment, and repeat surgery of proximal humeral fractures in the elderly. The Journal of Bone and Joint Surgery American Volume. 2011;93(2):121-131. doi: 10.2106/JBJS.I.01505.

5. Aaron D, Shatsky J, Paredes JC, Jiang C, Parsons BO, Evan L. Flatow, et al. Proximal humeral fractures: Internal fixation. Journal of Bone and Joint Surgery. 2012;94(24):2280-2288.

6. Terry GC, Chopp TM. Functional anatomy of the shoulder. Journal of Athletic Training. 2000;35(3):248-255.

7. Gerber C, Schneeberger A \& Vinh T. The arterial vascularization of the humeral head: An anatomical study. The Journal of Bone and Joint Surgery American Volume. 1990;72(10):1486-1494.

8. Laing P. The arterial supply of the adult humerus. The Journal of Bone and Joint Surgery American Volume. 1956;38(5):1105-1116.

9. Hettrich CM, Boraiah S, Dyke JP, Neviaser A, Helfet DL, Lorich DG, et al. Quantitative assessment of the vascularity of the proximal part of the humerus. The Journal of Bone and Joint Surgery American Volume. 2010;92(4):943-948. doi: 10.2106/JBJS.H.01144.

10. Crosby LA, Finnan RP, Anderson CG, Gozdanovic J, Miller MW. Tetracycline labeling as a measure of humeral head viability after 3or 4-part proximal humerus fracture. Journal of Shoulder and Elbow Surgery. 2009;18(6):851-858. doi: 10.1016/j.jse.2008.12.016.

11. Gardner MJ, Griffith MH, Dines JS, Briggs SM, Weiland AJ, Lorich DG. The extended anterolateral acromial approach allows minimally invasive access to the proximal humerus. Clinical Orthopaedics and Related Research. 2005;434:123-129.

12. Khmelnitskaya E, Lamont LE, Taylor SA, Lorich DG, Dines DM, David M. Dines, et al. Evaluation and management of proximal humerus fractures. Advances in Orthopedics. 2012;2012:1-10.

13. Khan L, Robinson C, Will E, Whittaker R. Assessment of axillary nerve function and functional outcome after fixation of complex proximal humeral fractures using the extended deltoid-splitting approach. Injury. 2009;40(2):181-185. doi: 10.1016/j.injury.2008.05.031. 
14. Buecking B, Mohr J, Bockmann B, Ruchholtz S. Deltoid-split or deltopectoral approaches for the treatment of displaced proximal humeral fractures? Clinical Orthopaedics and Related Research. 2014;472(5):1576-1585. doi: 10.1007/s11999-013-3415-7.

15. Flatow E, Bigliani L. Tips of the trade. Locating and protecting the axillary nerve in shoulder surgery: The tug test. Orthopaedic Review. 1992;21(4):503-505.

16. Rabi S, Evaniew N, Sprague S, Bhandari M \& Slobogean GP. Operative vs non-operative management of displaced proximal humerus fractures in the elderly: A systematic review and meta-analysis of randomized controlled trials. World Journal of Orthopedics. 2015;6(10):838-846. doi: 10.5312/wjo.v6.i10.838

17. Vachtsevanos L, Hayden L, Desai AS, Dramis A. Management of proximal humerus fractures in adults. World Journal of Orthopedics. 2014;5(5):685-693. doi: 10.5312/wjo.v5.i5.685.

18. Gulabi D, Cecen G, Bekler H, Saglam F, Tanju N. A study of 60 patients with percutaneous trigger finger releases: Clinical and ultrasonographic findings. Journal of Hand Surgery European Volume. 2014;39(7):699-703. doi: 10.1177/1753193413517992.

19. Krill M, Peck E. Percutaneous ultrasound-guided aspiration of an anterior cruciate ligament ganglion cyst: Description of technique and case presentation. PM\&R. 2014;6(12): 1166-1169. doi:10.1016/j. pmrj.2014.07.008.

20. Jaberg H, Warner J, Jakob R. Percutaneous stabilization of unstable fractures of the humerus. The Journal of Bone and Joint Surgery American Volume. 1992;74(4):508-515.

21. Keener J, Parsons B, Flatow E, Rogers K, Williams G, Galatz LM Outcomes after percutaneous reduction and fixation of proximal humerus fractures. Journal of Shoulder and Elbow Surgery. 2007;16(3):330-338. DOI:10.1016/j.jse.2006.09.006.

22.Zhu Y, Lu Y, Shen J, Zhang J, Jiang C. Locking intramedullary nails and locking plates in the treatment of two-part proximal humeral surgical neck fractures: A prospective randomized trial with a minimum of three years of follow-up. The Journal of Bone and Joint Surgery American Volume. 2011;93(2):159-168. doi: 10.2106/JBJS.J.00155.

23. Bernard J, Charalambides C, Aderinto J, Mok D. Early failure of intramedullary nailing for proximal humeral fractures. Injury. 2000;31(10):789-792.
24. Verbruggen J, Stapert J. Humeral fractures in the elderly: Treatment with a reamed intramedullary locking nail. Injury. 2007;38(8): 945953.

25. Katthagen J, Schwarze M, Meyer-Kobbe J, Voigt C, Hurschler C, Lill H. Biomechanical effect of calcar screws and bone block augmentation on medial support in locked plating of proximal humeral fractures. Clinical Biomechanics. 2014;29:735-741. doi: 10.1016/j. clinbiomech.2014.06.008.

26. Neviaser A, Hettrich C, Beamer B, Dines J, Lorich D. Endosteal strut augment reduces complications associated with proximal humerus locking plates. Clinical Orthopaedics and Related Research. 2011;469(12):3300-3306. doi: 10.1007/s11999-011-1949-0.

27. Badman B, Mighell M. Fixed-angle locked plating of two-, three-, and four-part proximal humerus fractures. The Journal of the American Academy of Orthopaedic Surgeons. 2008;16(5):294-302.

28. Sproul R, Iyengar J, Devcic Z, Feeley B. A systematic review of locking plate fixation of proximal humerus fractures. Injury. 2011;42(4):408413. doi:10.1016/j.injury.2010.11.058.

29. Clavert P, Adam P, Bevort A, Bonnomet F, Kempf J. Pitfalls and complications with locking plate for proximal humerus fracture. Journal of Shoulder and Elbow Surgery. 2010;19(4):489-494. doi: 10.1016/j.jse.2009.09.005.

30. Jost B, Spross C, Grehn H, Gerber C. Locking plate fixation of fractures of the proximal humerus: Analysis of complications, revision strategies and outcome. Journal of Shoulder and Elbow Surgery. 2013;22(4):542549. doi: 10.1016/j.jse.2012.06.008.

31. Bhandari M, Matthys G, Mckee M. Four part fractures of the proximal humerus. Journal of Orthopaedic Trauma. 2004;18(2): 126-127.

32. Simovitch R, Zumstein M, Lohri E, Helmy N, Gerber C. Predictors of scapular notching in patients managed with the Delta III reverse total shoulder replacement. The Journal of Bone and Joint Surgery American Volume. 2007;89(3):588-600.

33. Strassmair M, Jonas M, Schafer W, Palmer A. Distal radial fracture management with an intramedullary cage and fragment fixation. The Journal of Hand Surgery. 2016;41(8):833-840. doi: 10.1016/j. jhsa.2016.05.008. 